\title{
Reverse puncture device technique: an innovation of esophagojejunostomy in radical laparoscopic total gastrectomy
}

\author{
Xiaoxu Huang ${ }^{1}$, Li Xu${ }^{1}$, Hui Peng ${ }^{2}$, Hao Hu${ }^{1}$, Yan Jin ${ }^{1}$, Dayong Sun ${ }^{3}$, Kaifeng Hu${ }^{1}$ \& Yabin \\ Xia*, 1 \\ ${ }^{1}$ Department of Gastrointestinal Surgery, the First Affiliated Yijishan Hospital of Wannan Medical College, Wuhu, Anhui, PR China \\ ${ }^{2}$ Administration Office of Hospital Admission \& Discharge, The First Affiliated Yijishan Hospital of Wannan Medical College, Wuhu, \\ Anhui, PR China \\ ${ }^{3}$ Department of General Surgery, People's Hospital of Linquan County, Fuyang, Anhui, PR China \\ *Author for correspondence: Tel.: +86186055 31143; xiayabin1971@163.com
}

\begin{abstract}
Aim: To evaluate the feasibility, safety, short- and long-term efficacy of a reverse puncture device (RPD) technique for esophagojejunostomy in laparoscopic-assisted total gastrectomy. Patients \& methods: This retrospective study analyzed outcome data of 104 patients in propensity score matching whom were divided into the RPD and the purse-string suture technique group. Results: The RPD group had a shorter anvil placement time, shorter operative time, longer resected esophageal length, shorter incision length, shorter postoperative drainage time, shorter postoperative hospital stay and anastomotic complications than the purse-string suture technique group $(\mathrm{p}<0.05)$. Multivariate analysis showed that BMI (odds ratio: 6.285, 1.446-27.322) and anvil placement time (odds ratio: 5.645, 1.089-29.321) were independent risk factors for anastomotic complications $(p<0.05)$. Conclusion: Laparoscopic-assisted total gastrectomy using an RPD technique is feasible, safe and effective.
\end{abstract}

First draft submitted: 6 November 2018; Accepted for publication: 28 June 2019; Published online: 25 July 2019

Keywords: complication • gastric cancer • laparoscopic surgery • propensity score matching • reverse puncture device technique

Laparoscopy is a technological breakthrough that has led to faster recovery for a number of surgical disease processes including gastric cancer (GC) [1]. In 2002, Kanaya et al. reported total laparoscopic reconstruction of the digestive tract with radical gastrectomy for distal GC [2]. With the improvement in staplers and anastomotic techniques, total laparoscopic digestive tract reconstruction is becoming increasingly popular [3-5]. At present, Roux-en-Y anastomosis is one of the main methods for digestive tract reconstruction after radical laparoscopic-assisted total gastrectomy (LATG) [6,7]. However, the insertion of the anvil of a circular stapling device into the esophageal stump is a technically difficult, complicated procedure [8]. In 2009, Omori et al. [9] reported a simple and safe circular stapler insertion method using a reverse puncture device (RPD) for esophagojejunostomy in a hemidouble stapling technique during LATG. Over nearly a decade, relevant reports regarding anvil placement in the esophageal stump were published in succession [10-17]. In conventional open approaches, esophagojejunostomy has usually been performed using the purse-string suture technique (PST). Therefore, some scholars imitated the laparotomy method with the PST to complete intracorporeal circular-stapled esophagojejunostomy [18-22]. However, whether an RPD technique is superior to the traditional PST is not yet clear.

Previous retrospective studies provided the initial framework for the safety and short-term effectiveness of an RPD technique during LATG. However, additional research evaluating the RPD technique for GC seems warranted, especially with regard to the long-term efficacy. Therefore, for the first time, we performed long-term follow-up through more cases and aimed to obtain strong evidence of the feasibility and short- and long-term efficacy of the use of an RPD compared with PST for esophagojejunostomy after LATG with D2 lymph node dissection for GC. At the same time, the selection bias was reduced by the propensity score matching (PSM) method.

Future Medicine 


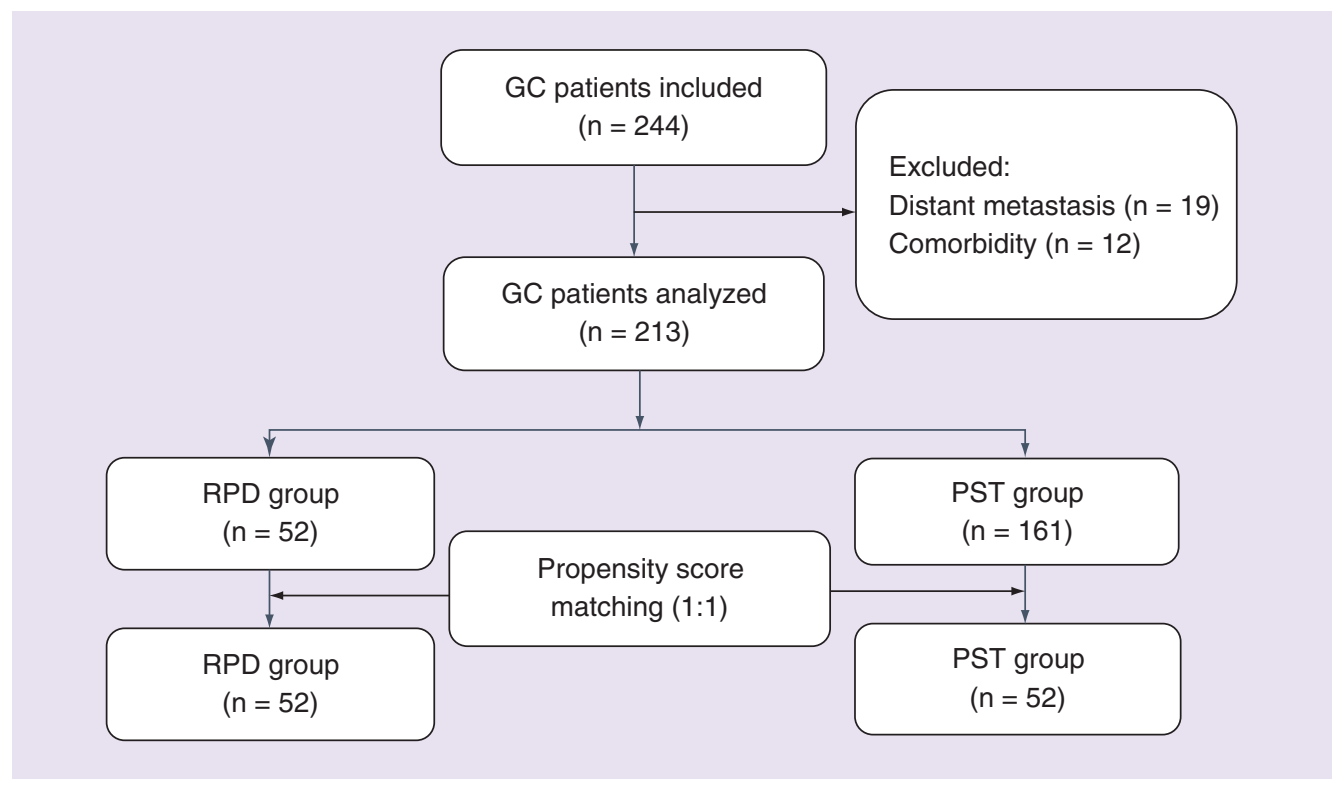

Figure 1. The flowchart of patient selection.

GC: Gastric cancer; PST: Purse-string suture technique; RPD: Reverse puncture device.

\section{Patients \& methods}

\section{General patient information}

In the retrospective analysis, data were collected from patients diagnosed of gastric adenocarcinoma at the First Affiliated Yijishan Hospital of Wannan Medical College Department of Gastrointestinal Surgery from June 2012 to December 2016. The case including criteria were as follows: gastroscopy and pathological biopsy performed before surgery, diagnosis of gastric adenocarcinoma; lesions located in the gastric body, fundus or esophagogastric junction or diffuse tumors; tumor clinical stage $\leq \mathrm{III}$ (according to the American Joint Committee on Cancer [AJCC] 7th edition Tumor, Node, Metastasis [TNM] tumor staging criteria); and postoperative pathological examination confirming gastric adenocarcinoma. The exclusion criteria were: distant metastasis by enhanced abdominal CT scanning; intraoperatively proved distant metastasis; patients with severe malnutrition, severe cardiopulmonary disease or coagulation dysfunction. Of the 244 patients with gastric adenocarcinoma who met the inclusion criteria, 31 patients were excluded for the following reasons: 19 patients proved distant metastasis intraoperative (15 patients had peritoneal metastasis and four patients had pancreas invasion), 12 patients had severe comorbidity. Therefore, 213 patients were finally included in this study. Figure 1 shows the flowchart of patient selection. In the pre-operative conversation, we informed the patient and their family, the relevant anastomosis in the operation. The advantages and disadvantages of the esophagojejunostomy reconstruction method using an RPD technique or a PST were discussed with the patients before surgery, and the procedure that was performed was selected by the patients. All patients signed informed consent before surgery. We also put the Chinese version (photograph) and translated version of the surgical informed consent in the Supplementary Materials. This study was approved by the ethics committee of Yijishan Hospital at Wannan Medical College.

\section{Surgical methodology}

The patient was placed in a supine position with the legs open and a mirror-holder between the legs. The surgeon stood on the left side, and the first assistant stood on the right of the operating table. General anesthesia with endotracheal intubation was administered. A Veress puncture needle was used to establish a pneumoperitoneum through a $10-\mathrm{mm}$ incision at the umbilicus, sustaining an abdominal pressure of up to $14 \mathrm{mmHg}(1 \mathrm{mmHg}=0.133 \mathrm{KPa})$. A five-port technique was used (Figure 2): a 10-mm trocar was placed in the umbilical region as the observation port. Three 5-mm trocars were placed beside the right rectus abdominus and left rectus abdominus and $6 \mathrm{~cm}$ proximal to these locations. A 12-mm trocar was placed $2 \mathrm{~cm}$ below the left rib edge at the front of the axilla, and the laparoscope and corresponding instrument were also placed in this area. Radical total gastrectomy and D2 lymph node dissection were performed after exploratory surgery. A 25-mm circular stapling device was used for 


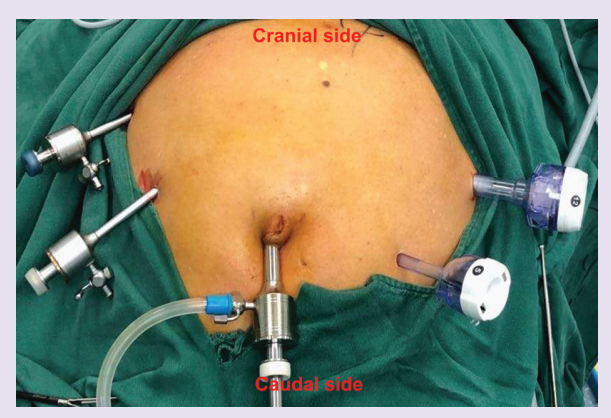

Figure 2. Port location. A $10-\mathrm{mm}$ trocar was placed on the umbilical region as the observation port. Beside the right rectus abdominis at the umbilical level, $6 \mathrm{~cm}$ above here, beside the left rectus abdominis at the umbilical level, three $5 \mathrm{~mm}$ trocar were placed at each position. $12 \mathrm{~mm}$ trocar was placed $2 \mathrm{~cm}$ below the left rib edge at axillary frontline level, the laparoscope and that correspond instrument are respectively placed.

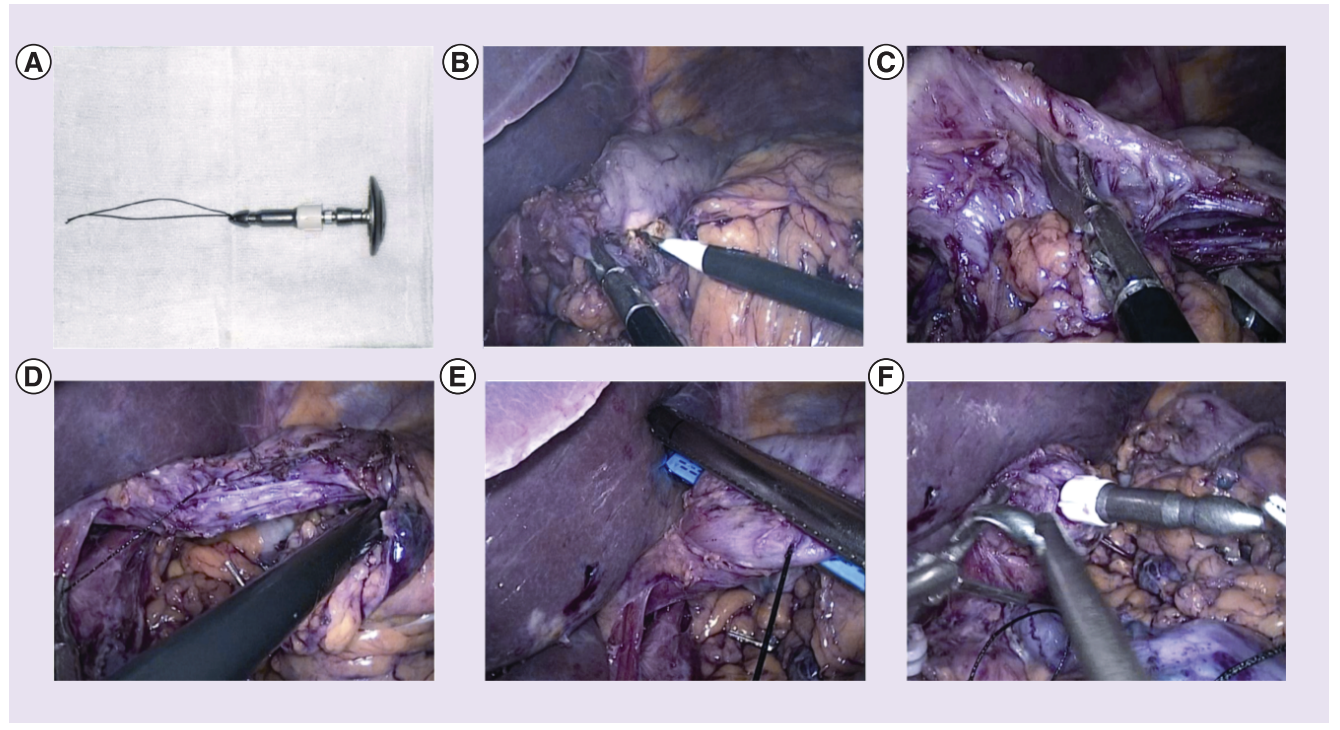

Figure 3. Surgical procedures of reverse puncture device technique. (A) The RPD production. The body is a $25 \mathrm{~mm}$ circular stapler anvil (Dst EEA, Medtronic corp), with its trocar secured with a 2-0 monofilament suture at the hole of the tip, and the suture tail knotted to form a woven braid. (B) $2-3 \mathrm{~cm}$ longitudinal incision of the anterior wall of the stomach at the cardia was performed. (C) The anvil with the silk thread was completely placed into the esophagus via the incision. (D) Pulled the silk thread out of the esophagus at the highest point of the esophageal incision. (E) The esophagus was transected at the precutting line. (F) The anvil was taken out at the reserved esophageal notch.

esophagojejunostomy in both groups (Dst EEA circular stapler, Medtronic Corp, MN, USA). In the PST group, conventional esophagojejunostomy was performed. The method used in this group was as follows: a small incision was made in the upper abdomen to enter the abdominal cavity. The PST was used at the lower end of the esophagus by using a purse-string clamp. The anvil of esophageal stump was inserted into the residual end of the esophagus, tightened and ligated with a purse string. The main body of the tubular stapler was placed in the distal end of the jejunum. Laparoscopic esophagojejunostomy was performed after the re-establishment of a pneumoperitoneum. A tubular stapler was then inserted into the distal jejunum, and the central rod was connected with the anvil after penetrating through the intestinal wall to complete the anastomosis. The detailed methods used in the RPD group (Figure 3) were as follows: a $4-\mathrm{cm}$ silk thread was inserted into a small hole at the tip of the connecting rod behind the anvil in advance, the silk thread was knotted and fixed, and the stapler anvil was placed through the $12-\mathrm{mm}$ trocar on the left side. A $2-3 \mathrm{~cm}$ longitudinal incision was made in the anterior wall of the stomach at the cardia to reach the esophagus, ensuring that the high point of the incision was positioned at the precut line. The anvil with the silk thread was completely inserted into the esophagus, and the silk thread was pulled out of the esophagus at the most proximal point of the esophageal incision. Approximately $0.3-0.5 \mathrm{~cm}$ of the esophageal notch was reserved at the position where the thread exited the anvil. Through this notch, the anvil was pulled out by the thread to achieve the insertion process under total laparoscopy. The main body of the tubular stapler was placed in the distal end of 
the jejunum. The detail of this procedure was shown in the video of applying RPD technology in LATG (video link https://youtu.be/M_3WQVZCffk). Laparoscopic esophagojejunostomy was performed after the re-establishment of a pneumoperitoneum. In both groups, the duodenum was resected with a disposable gastrointestinal (GI) cartridge (Echelon 60; Ethicon Endo-Surgery) under laparoscopy. Gastric specimens were removed through an upper median abdominal incision in PST group, and through a small incision around the umbilicus in RPD group. The mesentery was cut $15 \mathrm{~cm}$ below the Treitz ligament, and the vascular arch was ligated to free the distal jejunum. The proximal and distal jejunum were anastomosed $40 \mathrm{~cm}$ below the esophageal-jejunal anastomotic stoma. A disposable gastrointestinal (GI) cartridge (Echelon 60; Ethicon Endo-Surgery) was applied to close the distal jejunal stump. All surgeries were performed by the same team. The surgeons who participated in the trial had performed at least 200 laparoscopic radical gastrectomy procedures. We removed the first 20 patients who were placed in the circular stapler by the RPD method. The selected cases have successfully passed the surgeon's learning curve. The two groups of patients we enrolled at the same time. In addition to the difference in placement methods of anvil during the operation, the rest of the surgical procedures were consistent, and these videos were recorded. We also have strict uniform quality control.

\section{Observed indicators}

The anvil placement time was defined in the RPD group the time on the surgical video from when the anvil was placed into the abdominal cavity to the completion of placement, and in the PST group, it was defined as the intraoperative time from when the purse-string clamp entered the abdominal cavity until the anvil was placed. The operation time, intraoperative blood loss as measured by an aspirator and time to first flatus were recorded. The postoperative drainage time was measured, and indications for removal were drainage flow $<20 \mathrm{ml} / \mathrm{d}$, normal drainage fluid color, and no obvious symptoms of abdominal infection. Postoperative hospital stay was recorded until discharge standards including consumption of a semifluid diet, no requirement for intravenous fluid support, and ability to walk freely were met. Postoperative complications were as follows: anastomotic complications (including anastomotic leakage, anastomotic bleeding and anastomotic stenosis), abdominal bleeding, GI bleeding, abdominal infection, ileus, incisional infection, urinary tract infection, pulmonary infection and deep venous thrombosis. Other digestive tract leakage included leakage from the jejunal-jejunal anastomotic stoma and duodenal stump leakage.

\section{Follow-up}

Patients were followed-up every 3 months during the first 2 years and then every 6 months from 2 to 5 years. The survival time was defined as the period from the date of surgery to the date of death or final follow-up. All patients were monitored until death or June 2017, and the median follow-up time was 38 months (range: 3-60 months).

\section{Statistical analysis}

A logistic regression model was performed with 1:1 PSM adjusting the following covariates: age, gender, BMI, lesion location, tumor diameter, histological differentiation, pTNM stage, carcinoembryonic antigen (CEA) level, postoperative chemotherapy and comorbidities. Continuous variables were expressed as the mean \pm standard deviation (SD). Nonparametric data were expressed by the median quartile M (P25, P75). Continuous and categorical variables were compared using independent samples t-test, Mann-Whitney $U$ test and the $\chi^{2}$ test or Fisher's exact test, respectively. Using the Kaplan-Meier method and log-rank test, the cumulative survival rate was calculated. Logistic regression analysis was used to determine independent risk factors associated with anastomotic complications. SPSS 17.0 software (SPSS, Inc., IL, USA) was used for statistical analysis. A two-tailed p-value $<0.05$ was considered statistically significant.

\section{Results}

Patient clinicopathological characteristics

The general clinicopathological characteristics are shown in Table 1, including those of all the patients $(\mathrm{n}=213)$ and the propensity score-matched patients $(\mathrm{n}=104)$. After PSM, there were no significant differences observed between the patients in the RPD and PST groups in terms of clinicopathological characteristics, such as age, gender, BMI, lesion location, tumor diameter, histological differentiation, pTNM stage and comorbidities ( $>0.05)$. 


\begin{tabular}{|c|c|c|c|c|c|c|}
\hline \multirow[t]{2}{*}{ Characteristic } & \multicolumn{3}{|c|}{ All patients } & \multicolumn{3}{|c|}{ Propensity-matched patients } \\
\hline & $\operatorname{RPD}(n=52)$ & PST $(n=161)$ & p-value & $\operatorname{RPD}(n=52)$ & PST $(n=52)$ & p-value \\
\hline Gender: & & & 0.762 & & & 0.676 \\
\hline - Male & 36 & 115 & & 36 & 34 & \\
\hline - Female & 16 & 46 & & 16 & 18 & \\
\hline$-\leq 25$ & 38 & 132 & & 38 & 36 & \\
\hline$->25$ & 14 & 29 & & 14 & 16 & \\
\hline Lesion location: & & & 0.013 & & & 0.952 \\
\hline - Cardia & 24 & 40 & & 24 & 25 & \\
\hline - Stomach body & 21 & 96 & & 21 & 21 & \\
\hline - Well & 12 & 77 & & 12 & 13 & \\
\hline - Moderate & 24 & 56 & & 24 & 22 & \\
\hline - Poor & 16 & 28 & & 16 & 17 & \\
\hline pT stage: & & & 0.075 & & & 0.743 \\
\hline$-\mathrm{T} 1$ & 3 & 24 & & 3 & 5 & \\
\hline$-\mathrm{T} 2$ & 10 & 42 & & 10 & 11 & \\
\hline$-\mathrm{T} 3$ & 14 & 46 & & 14 & 16 & \\
\hline$-\mathrm{T} 4$ & 25 & 49 & & 25 & 20 & \\
\hline pN stage: & & & 0.950 & & & 0.686 \\
\hline$-\mathrm{NO}$ & 15 & 51 & & 15 & 18 & \\
\hline$-I I I$ & 31 & 71 & & 31 & 24 & \\
\hline CEA (ng/ml): & & & 0.190 & & & 0.387 \\
\hline$-\leq 3$ & 39 & 105 & & 39 & 35 & \\
\hline$->3$ & 13 & 56 & & 13 & 17 & \\
\hline $\begin{array}{l}\text { Postoperative } \\
\text { chemotherapy: }\end{array}$ & & & 0.664 & & & 0.377 \\
\hline - Yes & 40 & 119 & & 40 & 36 & \\
\hline$-\mathrm{No}$ & 12 & 42 & & 12 & 16 & \\
\hline Comorbidities: & & & 0.490 & & & 1.000 \\
\hline -Yes & 40 & 116 & & 40 & 40 & \\
\hline- No & 12 & 45 & & 12 & 12 & \\
\hline
\end{tabular}

Intraoperative \& postoperative outcomes

The intraoperative and postoperative observation indexes for comparison of 104 patients in both groups are shown in Tables 2 and 3. The RPD group had a shorter anvil placement time (11.6 vs $13.3 \mathrm{~min}, \mathrm{p}=0.004)$, shorter operative time ( 218.6 vs $234.5 \mathrm{~min}, \mathrm{p}=0.002)$, longer resected esophageal length $(3.5$ vs $3.1 \mathrm{~cm}, \mathrm{p}=0.013)$, shorter incision length $(5.2$ vs $7.6 \mathrm{~cm}, \mathrm{p}=0.000)$, shorter postoperative drainage time ( 8.2 vs $9.9 \mathrm{~d}, \mathrm{p}=0.000)$ and shorter postoperative hospital stay ( 9.5 vs $12.0 \mathrm{~d}, \mathrm{p}=0.003)$ than the PST group. The amount of intraoperative 
Table 2. Comparison of intraoperative observation indexes of two groups.

\begin{tabular}{|lllll|}
\hline Characteristic & RPD group $(\mathbf{n}=52)$ & PST group $(\mathbf{n}=52)$ & t value & p-value \\
\hline Anvil placement time $(\mathrm{min})$ & $11.6 \pm 2.6$ & $13.3 \pm 3.3$ & -2.961 & 0.004 \\
\hline Operation time $(\mathrm{min})$ & $218.6 \pm 23.7$ & $234.5 \pm 27.8$ & -3.134 & 0.002 \\
\hline Hemorrhage amount $(\mathrm{ml})$ & $148.3 \pm 45.2$ & $166.3 \pm 48.1$ & -1.962 & 0.052 \\
\hline $\begin{array}{l}\text { Resected esophageal length } \\
(\mathrm{cm})\end{array}$ & $3.5(2.4-4.2)$ & $3.1(2.3-3.8)$ & & $0.013^{\dagger}$ \\
\hline Length of the incisions $(\mathrm{cm})$ & $5.2 \pm 0.8$ & $7.6 \pm 1.0$ & -12.932 & 0.000 \\
\hline
\end{tabular}

$\dagger$ Mann-Whitney p-value.

PST: Purse-string suture technique; RPD: Reverse puncture device.

\section{Table 3. Comparison of postoperative observation indexes of two groups.}

\begin{tabular}{llll} 
Characteristic & RPD group $(n=52)$ & PST group $(n=52)$ & t value \\
\hline Postoperative exhaust time (d) & $3.9 \pm 1.2$ & $3.9 \pm 1.5$ & -0.070 \\
\hline Postoperative drainage time (d) & $8.2 \pm 1.7$ & $9.9 \pm 2.3$ & -4.377 \\
\hline $\begin{array}{l}\text { Postoperative hospitalization } \\
\text { time (d) }\end{array}$ & $9.5(9.0-11.0)$ & $12.0(10.0-13.0)$ & 0.944 \\
\hline
\end{tabular}

$\dagger$ Mann-Whitney p-value.

PST: Purse-string suture technique; RPD: Reverse puncture device.

\begin{tabular}{|c|c|c|c|c|}
\hline Complications & RPD group $(n=52)$ & PST group $(n=52)$ & $\chi^{2}$ value & p-value \\
\hline Overall complications & $8(15.3 \%)$ & $12(23.0 \%)$ & 0.990 & 0.320 \\
\hline Anastomotic complications: & $2(3.8 \%)$ & $8(15.3 \%)$ & 3.983 & $0.046 / 0.092^{\dagger}$ \\
\hline - Anastomotic leakage & $2(3.8 \%)$ & $4(7.6 \%)$ & 0.177 & 0.674 \\
\hline - Anastomotic bleeding & $0(0 \%)$ & $3(5.7 \%)$ & 1.373 & 0.241 \\
\hline - Anastomotic stenosis & $0(0 \%)$ & $1(1.9 \%)$ & & $1.000^{\dagger}$ \\
\hline Digestive tract leakage (other) & $0(0 \%)$ & $1(1.9 \%)$ & & $1.000^{\dagger}$ \\
\hline Wound infection & $2(3.8 \%)$ & $2(3.8 \%)$ & 0.000 & 1.000 \\
\hline Abdominal bleeding & $1(1.9 \%)$ & $1(1.9 \%)$ & 0.000 & 1.000 \\
\hline Ileus & $1(1.9 \%)$ & $0(0 \%)$ & & $1.000^{\dagger}$ \\
\hline Pneumonia & $1(1.9 \%)$ & $0(0 \%)$ & & $1.000^{\dagger}$ \\
\hline Urinary tract infection & $1(1.9 \%)$ & $0(0 \%)$ & & $1.000^{\dagger}$ \\
\hline
\end{tabular}

blood loss and time to first flatus were not significantly different between the two groups ( $\mathrm{p}>0.05)$. Incision of RPD and PST patients were shown in Supplementary Figure 1.

\section{Postoperative complications}

The overall postoperative complication rates in the RPD and PST groups were 15.3 and $23 \%$, respectively; $\mathrm{p}=0.320$. In the RPD and PST groups, postoperative anastomotic complications occurred in two (3.8\%) and eight (15.3\%) patients, respectively $(\mathrm{p}=0.046 /$ Fisher $\mathrm{p}=0.092)$. The results are shown in Table 4. Regarding the details of postoperative complications, a total of seven cases of digestive tract leakage were confirmed by lipiodol radiography after surgery and were cured after treatment with peritoneal lavage combined with enteral and parenteral nutrition support for 7-12 days. Endoscopic hemostatic treatment cured three cases of anastomotic bleeding. One patient with esophageal and jejunal anastomotic stenosis was cured by means of balloon dilation. Patients suffering from abdominal hemorrhage and ileus were treated successfully with conservative methods. Pulmonary and incisional infections were cured with antimicrobial treatment and continuous changing of incisional dressings. There was no mortality in two groups during the perioperative period. 


\section{Univariate \& multivariate analyses of factors associated with anastomotic complications}

The univariate analysis showed that BMI and anvil placement time were closely related to anastomotic complications $(\mathrm{p}<0.05)$. Furthermore, in the multivariate analysis BMI ( $\mathrm{p}=0.014$; odds ratio: 6.285, 1.446-27.322) and anvil placement time ( $\mathrm{p}=0.040$; odds ratio: $5.645,1.089-29.321)$ were independent risk factors for anastomotic complications (Table 5).

\section{Survival after surgery}

The 5-year overall survival (OS) rate in the RPD group was not significantly different to that in the PST group (56.8 vs $54.7 \%, \mathrm{p}=0.629)$ (Supplementary Figure 2 ).

\section{Discussion}

Two main types of anastomosis are used in laparoscopic esophagojejunostomy: tubular anastomosis and linear anastomosis [7,23,24]. Tubular anastomosis has advantages in obtaining improved incisional margins, but it is technically difficult in obese people. Jeong et al. [25] reported in 2009 that the use of OrVil ${ }^{T M}$ had a good effect on patients with cardiac cancer with a high BMI or obesity. However, continuous improvements in laparoscopy techniques have provided new possibilities with regard to operative success in oncologic patients and the best chance for improved recovery during and after surgery. A clinical study introduced the reconstruction method of intracorporeal circular stapling esophagogastrostomy/esophagojejunostomy with an RPD and confirmed the feasibility and safety of this technique from a technical level [26]. In this study, the RPD technique in LATG was applied to explore the short- and long-term efficacy and safety compared with traditional placement of an anvil with the PST.

In this study, the background patient data of patients in the two groups were not comparable with that of the entire cohort; therefore, it could be argued that the direct comparison of these indexes is not appropriate for all patients because some factors, such as age, histological differentiation and tumor diameter, might be independently responsible for poor survival outcome, regardless of postoperative complications. Therefore, we employed PSM method to adjust for significant differences in the clinicopathologic characteristics of GC patients with aim to control the selection bias and underlying confounding factors on outcome.

In the present study, we showed that the RPD technique is significantly better than the traditional PST with regard to the intraoperative outcomes including placement time, operative time and esophageal resection length indicating that the RPD method allowed the anvil to be inserted quickly to complete the anastomosis, shortening the total operative time and allowing resection of a large amount of esophageal tissue to ensure improved incisal margins. The results of this study also showed that the RPD group had a significantly better postoperative recovery than the PST group with regard to factors such as abdominal cavity drainage time and length of postoperative hospitalization.

The effect of routine drainage after abdominal surgery with enteric anastomoses is controversial. In particular, the role of peritoneal drain placement after total gastrectomy for adenocarcinoma is not well established. Some scholars have shown peritoneal drain placement after total gastrectomy is associated with neither a decrease in the frequency and severity of adverse postoperative outcomes, including anastomotic leak and mortality, nor a decrease in the need for secondary drainage procedures or reoperation [27]. The successful management of anastomotic leak depends on early detection and prompt treatment. The presence of digestive fluids in surgical drains is unequivocal evidence. Some scholars believe that effective drainage is the most effective treatment in the early stage of postoperative anastomotic leak. Conservative treatment with drainage and nutritional support is the most common approach [28]. In this study, we had a long time to indwelling the drainage tube after surgery. Analysis of the relevant reasons, on the one hand, for safety reasons, the postoperative drainage tube removal of this cohort of patients was relatively conservative. On the other hand, these patients were older, with an average age of 64.5. In a retrospective evaluation of a large patient cohort, Tu et al. [29] developed a nomogram to predict the individual risk of anastomotic leakage. Patient age $\geq 65$ years and malnutrition were independently associated with the risk of leakage. We believe that timely flushing through the indwelling abdominal drainage when anastomotic leakage occurs is a simple and effective treatment.

In terms of postoperative complications, the reported incidence after laparoscopic radical gastrectomy for GC ranges from 7.3 to $23.3 \%$ [30,31]. In this study, the overall postoperative complication rates in the RPD and PST groups were 15.3 and 23\%, respectively, and there was no significant difference between the two groups. However, the total numbers of anastomotic complications, such as anastomotic leakage, bleeding, and 
Research Article Huang, Xu, Peng et al.

Table 5. Univariate and multivariate analyses of factors associated with anastomotic complications.

Characteristic

Univariate analysis

OR

$95 \% \mathrm{Cl}$

p-value

OR

Multivariate analysis

Age (years):

$-\leq 65$

$$
1.000
$$

$->65$

1.212

$0.321-4.576$

0.777

Gender:

- Male

1.000

- Female

0.484

$0.097-2.416$

0.377

BMI $\left(\mathrm{kg} / \mathrm{m}^{2}\right)$ :

$-\leq 25 \quad 1.000$

.000

1.000

Lesion location:

- Cardia $\quad 1.000$

- Stomach body $\quad 1.354$

- Diffuse type $\quad 1.263$

$\begin{array}{lll}1.137-16.833 & 0.032 & 6.285\end{array}$

$95 \% \mathrm{Cl}$

p-value

Tumor diameter $(\mathrm{mm})$ :

$-\leq 50$

1.000

$->50 \quad 0.774$

0.700

Histological differentiation:

- Well 1.000

- Moderate $\quad 1.813$

- Poor $\quad 0.161$

0.145-12.808

$\begin{array}{ll}0.129-12.416 & 0.963\end{array}$

pTNM stage:

$0.432-7.596$

0.017-1.514 0.099

$\begin{array}{ll}-1 & 1.000\end{array}$

- II 4.676

- III 5.521

0.720-30.365

$\begin{array}{ll}0.999-30.502 & 0.129\end{array}$

CEA (ng/ml):

- $\leq 3 \quad 1.000$

- >3 0.932

0.932

$0.256-4.419$

0.932

Comorbidities:

- Yes $\quad 1.000$

- No $\quad 0.818$

$0.162-4.140 \quad 0.808$

Method of performing anvil:

- RPD 1.000

- PST 4.545

Anvil placement time ( $\mathrm{min})$ :

$-\leq 12 \quad 1.000$

- $>12 \quad 6.162$

Operation time (min):

- $\leq 220 \quad 1.000$

$->220 \quad 4.744$

Hemorrhage amount (ml):

$-\leq 150$

1.000

- >150 4.545

$0.916-22.549$

0.064

Resected esophageal length $(\mathrm{cm})$ :

$\begin{array}{llll}-\leq 3.5 & 1.000 & & \\ ->3.5 & 0.421 & 0.085-2.098 & 0.291\end{array}$

OR: Odds ratio; PST: Purse-string suture technique; RPD: Reverse puncture device. 
stenosis, was significantly lower in the RPD group than in the PST group. These complications may benefit from reduced surgical invasiveness, and bleeding during surgery and inflammatory responses are associated with the anvil placement procedure. Effective measures must be taken to avoid the occurrence postoperative complications in LATG, especially anastomotic complications, not only in the interests of improving short-term outcomes, but also for improving the long-term prognosis. Therefore, identifying the associated risk factors is important for minimizing the incidence of postoperative complications. We converted the continuous variables into categorical variables and performed univariate and multivariate analyses in the cohort to investigate risk factors correlated with anastomotic complications. The multivariate analysis revealed that higher BMI and longer anvil placement time were independent risk factors for the occurrence of anastomotic complications. Extensive studies have reported that BMI is a significant risk factor for postoperative complications, which is also consistent with our results [32-34]. Patients with a higher BMI had increased postoperative anastomotic complications rates. Therefore, more attention needs to be paid to patients with a higher BMI during preoperative assessment and postoperative management, and perioperative bleeding must be reduced.

In contrast to the relatively complicated method of inserting the anvil with the PST, the RPD technique is simpler and was first applied to LATG. As reported previously [9], the RPD method involves a suture with a needle passing through a small hole at the top of a circular stapler anvil that is then knotted and fixed. During this process, a small incision is made in the front wall of the esophagus. Then, the anvil is completely fed into the lower esophageal cavity through the incision, and a suture needle is used in reverse to penetrate the front wall of the esophagus $1 \mathrm{~cm}$ above the incision. The anvil is withdrawn and tightened, and a linear cutting and closing device is used to close the residual end of the esophagus below the nail seat. There is a risk of secondary injury when the suture needle enters the abdominal cavity. Based on this method, we improved the procedure by removing the suture needle and avoiding a second esophageal incision; the upper edge of the tumor should be accurately marked during surgery to confirm the esophageal precut line. We eliminated the suturing procedure to simplify the surgery. Thus, this modified technique is safer, more efficient and shortens the operative time.

However, there are still several limitations in our study. First, this is a retrospective study, and the conclusions have yet to be further confirmed by a large-sample, prospective randomized controlled trial. Second, although we decreased the selection bias by using PSM method, still there is systematic bias. Therefore, there is a clearly a critical need to perform a large-sample, prospective randomized controlled trial to characterize the efficacy of the RPD technique, so that the most suitable anvil insertion method can be evaluated accurately.

\section{Conclusion}

The application of the RPD technique for digestive tract reconstruction in LATG proved to be safe and feasible. Our research has shown good curative effects and provided a new method of laparoscopic digestive tract reconstruction.

Supplementary data

To view the supplementary data that accompany this paper please visit the journal website at: www.futuremedicine.com/doi/sup $\mathrm{pl} / 10.2217 /$ fon-2018-0837

\section{Authors' contributions}

YB Xia and XX Huang conceived of the study and data collection. XX Huang, L Xu and H Peng carried out the data analysis. XXH, $\mathrm{LX}, \mathrm{H}$ Hu and $Y$ Jin drafted the manuscript. DY Sun and KF Hu participated in the critical revision. All authors read and approved the final manuscript.

\section{Acknowledgments}

The authors thank the patients, their families, the investigators and staff who participated in the study. Medical writing assistance was provided by Gretchen $V$ and Sarah Conte, American Journal Experts Corp. We thank them for their help.

\section{Financial \& competing interests disclosure}

Anhui Provincial University Science Research Project, grant numbers: KJ2018A0247, KJ2016A733 and KJ2017A272; Wannan Medical College Cultivation Fund of the Key Scientific Project, grant number: WK2017ZF10; and Anhui Provincial University Social Research Project, grant number: SK2017A0214. The authors have no other relevant affiliations or financial involvement with any organization or entity with a financial interest in or financial conflict with the subject matter or materials discussed in the manuscript apart from those disclosed. 
Medical writing assistance was provided by American Journal Experts Corp. This assistance was funded by Anhui Provincial University Science Research Project, grant number: KJ2018A0247.

Ethical conduct of research

The authors state that this study was approved by the Ethics Committee of Yijishan Hospital at Wannan Medical College and have followed the principles outlined in the Declaration of Helsinki for all human or animal experimental investigations. In addition, for investigations involving human subjects, informed consent has been obtained from the participants involved.

\section{Open access}

This work is licensed under the Creative Commons Attribution-NonCommercial-NoDerivatives 4.0 Unported License. To view a copy of this license, visit: http://creativecommons.org/licenses/by-nc-nd/4.0/

\section{Summary points}

- At present, Roux-en-Y anastomosis is one of the main methods for digestive tract reconstruction after radical laparoscopic-assisted total gastrectomy (LATG).

- However, the insertion of the anvil of a circular stapling device into the esophageal stump is a technically difficult, complicated procedure.

- We now report a feasible, safe and efficacy circular stapler insertion method using a reverse puncture device (RPD) for esophagojejunostomy in a hemidouble stapling technique during LATG compared with the conventional purse-string suture technique (PST).

- A total of 213 gastric cancer (GC) patients who underwent LATG from June 2012 to December 2016 were retrospectively analyzed. Among them, 104 patients were divided by propensity score matching according to the method by which an anvil was placed in the esophageal stump into the RPD and PST group.

- The RPD group had a shorter anvil placement time (11.6 vs $13.3 \mathrm{~min}, \mathrm{p}=0.004$ ), shorter operative time (218.6 vs $234.5 \mathrm{~min}, \mathrm{p}=0.002)$, longer resected esophageal length $(3.5 \mathrm{vs} 3.1 \mathrm{~cm}, \mathrm{p}=0.013)$, shorter incision length $(5.2$ vs $7.6 \mathrm{~cm}, \mathrm{p}=0.000$ ), shorter postoperative drainage time (8.2 vs $9.9 \mathrm{~d}, \mathrm{p}=0.000)$ and shorter postoperative hospital stay ( 9.5 vs $12.0 \mathrm{~d}, \mathrm{p}=0.003$ ) than the PST group.

- In the RPD and PST groups, postoperative anastomotic complications occurred in two (3.8\%) and eight (15.3\%) patients, respectively $(p=0.046 /$ Fisher $p=0.092)$. Multivariate analysis showed that BMI $(p=0.014$; odds ratio: $6.285,1.446-27.322)$ and anvil placement time $(p=0.040$; odds ratio: $5.645,1.089-29.321)$ were independent risk factors for anastomotic complications.

- The application of the RPD technique for digestive tract reconstruction in LATG proved to be safe and feasible.

- Our research has shown good curative effects and provided a new method of laparoscopic digestive tract reconstruction.

\section{References}

Papers of special note have been highlighted as: $\bullet$ of interest; $\bullet \bullet$ of considerable interest

1. Ward MA, Ujiki MB. Creation of a jejunal pouch during laparoscopic total gastrectomy and Roux-en-Y esophagojejunostomy. Ann. Surg. Oncol. 24(1), 184-186 (2017).

2. Kanaya S, Gomi T, Momoi $\mathrm{H}$ et al. Delta-shaped anastomosis in totally laparoscopic Billroth I gastrectomy: new technique of intraabdominal gastroduodenostomy. J. Am. Coll. Surg. 195(2), 284-287 (2002).

- Reports total laparoscopic reconstruction of the digestive tract with radical gastrectomy for distal gastric cancer.

3. Nakauchi M, Suda K, Kadoya S, Inaba K, Ishida Y, Uyama I. Technical aspects and short- and long-term outcomes of totally laparoscopic total gastrectomy for advanced gastric cancer: a single-institution retrospective study. Surg. Endosc. 30(10), 4632-4639 (2016).

4. Huang ZN, Huang $\mathrm{CM}$, Zheng $\mathrm{CH}$ et al. Digestive tract reconstruction using isoperistaltic jejunum-later-cut overlap method after totally laparoscopic total gastrectomy for gastric cancer: short-term outcomes and impact on quality of life. World J. Gastroenterol. 23(39), 7129-7138 (2017).

5. Chen YS, Wu SD, Kong J. Transumbilical single-incision laparoscopic subtotal gastrectomy and total intracorporeal reconstruction of the digestive tract in the treatment of benign peptic ulcers. J. Surg. Res. 192(2), 421-425 (2014).

6. Takahashi M, Terashima M, Kawahira $\mathrm{H}$ et al. Quality of life after total vs distal gastrectomy with Roux-en-Y reconstruction: use of the postgastrectomy syndrome assessment scale-45. World J. Gastroenterol. 23(11), 2068-2076 (2017).

7. Ma JJ, Zang L, Yang A et al. A modified uncut Roux-en-Y anastomosis in totally laparoscopic distal gastrectomy: preliminary results and initial experience. Surg. Endosc. 31(11), 4749-4755 (2017).

8. Takiguchi S, Sekimoto M, Fujiwara Y et al. A simple technique for performing laparoscopic purse-string suturing during circular stapling anastomosis. Surg. Today 35(10), 896-899 (2005). 
9. Omori T, Oyama T, Mizutani S et al. A simple and safe technique for esophagojejunostomy using the hemidouble stapling technique in laparoscopy-assisted total gastrectomy. Am. J. Surg. 197(1), e13-e17 (2009).

-. It was first time reported a simple and safe circular stapler insertion method using a reverse puncture device (RPD) for esophagojejunostomy in a hemidouble stapling technique during laparoscopic-assisted total gastrectomy.

10. Omori T, Moon JH, Yamamoto K et al. A modified efficient purse-string stapling technique (mEST) that uses a new metal rod for intracorporeal esophagojejunostomy in laparoscopic total gastrectomy. Transl. Gastroenterol. Hepatol. 2, 61 (2017).

11. Li X, Hong L, Ding D et al. Comparison of OrVil and RPD in laparoscopic total gastrectomy for gastric cancer. Surg. Endosc. 31(11), 4773-4779 (2017).

12. Ali B, Park CH, Song KY. Intracorporeal esophagojejunostomy using hemi-double-stapling technique after laparoscopic total gastrectomy in gastric cancer patients. Ann. Surg. Treat. Res. 92(1), 30-34 (2017).

13. Wang H, Hao Q, Wang $\mathrm{M}$ et al. Esophagojejunostomy after laparoscopic total gastrectomy by OrVil or hemi-double stapling technique. World J. Gastroenterol. 21(29), 8943-8951 (2015).

14. Kosuga T, Hiki N, Nunobe S et al. Does the single-stapling technique for circular-stapled esophagojejunostomy reduce anastomotic complications after laparoscopic total gastrectomy? Ann. Surg. Oncol. 22(11), 3606-3612 (2015).

15. Amisaki M, Kihara K, Endo K et al. Comparison of single-stapling and hemi-double-stapling methods for intracorporeal esophagojejunostomy using a circular stapler after totally laparoscopic total gastrectomy. Surg. Endosc. 30(7), 2994-3000 (2015).

16. Chen DL, Ding D, Ke ZW. [Clinical application of reverse puncture device(RPD) in laparascopic esophagogastrectomy (esophagojejunostomy): a report of 18 cases]. Zhonghua Wei Chang Wai Ke Za Zhi 16(10), 956-959 (2013).

17. Shim JH, Yoo HM, Oh SI et al. Various types of intracorporeal esophagojejunostomy after laparoscopic total gastrectomy for gastric cancer. Gastric Cancer 16(3), 420-427 (2012).

18. Liu W, Guo Y, Qiu Z, Niu D, Zhang J. Intracorporeal circular stapled esophagojejunostomy using conventional purse-string suture instrument after laparoscopic total gastrectomy. J. Laparoendosc. Adv. Surg. Tech. A 27(12), 1299-1304 (2017).

19. Du J, Shuang J, Li J, Li J, Hua J. Intracorporeal circular-stapled esophagojejunostomy after laparoscopic total gastrectomy: a novel self-pulling and holding purse-string suture technique. J. Am. Coll. Surg. 218(3), e67-e72 (2014).

20. Kong SH, Suh Y, Kwon S, Lee HJ, Kim HH, Yang HK. Stable purse-string suturing using an anterior esophagotomy for reconstruction with a circular stapler during laparoscopic total gastrectomy. Asian J. Endosc. Surg. 6(2), 82-89 (2013).

21. Kim HI, Cho I, Jang DS, Hyung WJ. Intracorporeal esophagojejunostomy using a circular stapler with a new purse-string suture technique during laparoscopic total gastrectomy. J. Am. Coll. Surg. 216(2), e11-e16 (2013).

22. Kinoshita T, Oshiro T, Ito K, Shibasaki H, Okazumi S, Katoh R. Intracorporeal circular-stapled esophagojejunostomy using hand-sewn purse-string suture after laparoscopic total gastrectomy. Surg. Endosc. 24(11), 2908-2912 (2010).

23. Yang H, Xing J, Cui M et al. [Efficacy evaluation of laparoscopy-assisted radical gastrectomy in obese patients with gastric cancer]. Zhonghua Wei Chang Wai Ke Za Zhi 17(8), 776-780 (2014).

24. Yurtcu M, Abasiyanik A, Arbag H, Oz M. An oblique anastomosis has more linear length than a transverse anastomosis of a tubular structure in oesophageal anastomosis. Pediatr. Surg. Int. 25(2), 163-167 (2009).

25. Jeong O, Park YK. Intracorporeal circular stapling esophagojejunostomy using the transorally inserted anvil (OrVil) after laparoscopic total gastrectomy. Surg. Endosc. 23(11), 2624-2630 (2009).

-• It was first time reported that the use of OrVilTM had a good effect on patients with cardiac cancer with a high BMI or obesity.

26. Chen D, Cheng P, Ding D, Ke Z. Feasibility and safety of a novel reverse puncture device (RPD) for laparoscopic esophagogastrostomy/esophagojejunostomy. Int. J. Clin. Exp. Med. 7(9), 2497-2503 (2014).

27. Dann GC, Squires MH 3rd, Postlewait LM et al. Value of peritoneal drain placement after total gastrectomy for gastric adenocarcinoma: a multi-institutional analysis from the US gastric cancer collaborative. Ann. Surg. Oncol. 22(Suppl. 3), S888-S897 (2015).

28. Makuuchi R, Irino T, Tanizawa Y, Bando E, Kawamura T, Terashima M. Esophagojejunal anastomotic leakage following gastrectomy for gastric cancer. Surg. Today 49(3), 187-196 (2019).

29. Tu RH, Lin JX, Zheng CH et al. Development of a nomogram for predicting the risk of anastomotic leakage after a gastrectomy for gastric cancer. Eur. J. Surg. Oncol. 43(2), 485-492 (2017).

30. Lee JH, Ahn SH, Park DJ, Kim HH, Lee HJ, Yang HK. Laparoscopic total gastrectomy with D2 lymphadenectomy for advanced gastric cancer. World J. Surg. 36(10), 2394-2399 (2012).

31. Hosono S, Arimoto Y, Ohtani H, Kanamiya Y. Meta-analysis of short-term outcomes after laparoscopy-assisted distal gastrectomy. World J. Gastroenterol. 12(47), 7676-7683 (2006).

32. Li Z, Bai B, Zhao Y et al. Severity of complications and long-term survival after laparoscopic total gastrectomy with D2 lymph node dissection for advanced gastric cancer: a propensity score-matched, case-control study. Int. J. Surg. 54(Pt A), 62-69 (2018).

33. Lin YS, Huang KH, Lan YT et al. Impact of body mass index on postoperative outcome of advanced gastric cancer after curative surgery. J. Gastrointest. Surg. 17(8), 1382-1391 (2013).

34. STARSurg Collaborative. Multicentre prospective cohort study of body mass index and postoperative complications following gastrointestinal surgery. Br. J. Surg. 103(9), 1157-1172 (2016).

-• Reports that BMI is a significant risk factor for postoperative complications following gastrointestinal surgery. 
